Author version: Mar. Geod., vol.35(4); 2012; 441-454

\title{
A simple method to minimize orientation effects in a profiling radiometer
}

\author{
T.Suresh, Madhubala Talaulikar, Elgar Desa, S.G. Prabhu Matondkar, T. Srinivasa Kumar*, \\ Aneesh Lotlikar* \\ National Institute of Oceanography, Goa, India \\ *Indian National Centre for Ocean Information and Services, Hyderabad, India \\ Email: $\underline{\text { suresh@nio.org }}$
}

\begin{abstract}
Marine optical parameters required for ocean color satellite applications need to be measured with high accuracy and errors within the permissible limits. These stringent requirements demand careful measurements of optical parameters. Though the free fall radiometer is found to be a better option for measuring underwater light parameters as it avoids the effects of ship shadow and is easy to operate, the measurements however demand profiling the radiometer vertical in water with minimum tilt. Here we present the results of our observations on the tilts of the radiometer from the measurements in the Arabian Sea. Since there is hardly any study carried-out on the tilt of the profiling radiometer, the result of this study will help in the better design of such marine instruments. The tilt of the radiometer near the surface of water is attributed to the mode of deployment and environment parameters, while the tilt at depth of water is influenced by the density variations of the water. Here we also demonstrate a method of deploying the instrument that minimize the tilt of the instrument at the surface layer of water.
\end{abstract}

Keywords : profiling radiometer, tilt, orientation, optical data, free falling body 


\section{Introduction}

One of the primary objectives of the radiometric measurements of underwater light for ocean color applications is to measure and derive spectral water-leaving radiance, $\mathrm{L}_{\mathrm{w}}(\lambda)$ with least uncertainty and high accuracy under all conditions. Solar light entering the water undergoes scattering and absorption processes by the constituents in water such as water molecules, dissolved and detrital materials, phytoplankton and suspended matters and hence there is a change in spectral characteristics of light in water. Thus the light emerging from the surface of water known as $L_{w}(\lambda)$, carries information about the optical properties of water and the optically active constituents in water. Downwelling solar irradiance, $\mathrm{E}_{\mathrm{d}}(\lambda)$ is defined as the amount of solar radiation per unit area, per wavelength $\left(\mathrm{W} / \mathrm{m}^{2} / \mathrm{nm}\right)$ and water leaving radiance is defined as the amount of light leaving the ocean surface per unit area, per wavelength and per solid angle $\left(\mathrm{W} / \mathrm{m}^{2} / \mathrm{nm} / \mathrm{sr}\right)$. Hence for ocean color studies, the properties of solar light available at the surface of water (downwelling solar irradiance) and also those emerging from the water (water leaving radiance) are essential optical parameters required to derive information about the constituents in water and they are often used to model or develop algorithms to derive various parameters such as chlorophyll, suspended matter, CDOM and other related parameters. $\mathrm{L}_{\mathrm{w}}(\lambda)$ and $\mathrm{E}_{\mathrm{d}}(\lambda)$ are measured using a spectral radiometer and this radiometer could be surface measuring instrument mounted on a platform above water or a profiling underwater radiometer(Mueller and Austin1995; Mueller et al. 2003; Friedman et al. 2003). The acceptable uncertainty in $\mathrm{L}_{\mathrm{w}}(\lambda)$ is less than $5 \%$ for satellite validation (Hooker and Esaias 1993; Hooker et al. 2002). In order to achieve the desired accuracy we need to have better understanding of the sources of errors and evolve solutions to avoid them.

There are two prime sources of errors from the measurements of optical parameters using a radiometer that has been studied extensively, one from the shadow of ship or super structure known as ship shadow and the other arising from the effect of wave focusing-defocusing (Hooker et al. 2003). Apart from these there are sources of errors from the instrument itself such as self-shading of the instrument (Gordon and Ding 1992; Zibordi and Ferrari 1995; Aas and Korsb 1997), dark value correction and temperature effects. The additional sources of errors which can be minimized arise from the mode of deployment, intermittent cloud patches during measurements; depth offset adjustment to determine the exact surface depth.

The surface values of apparent optical properties are largely affected by the ship shadow and among these the upwelling radiances, $\mathrm{L}_{\mathrm{u}}(\lambda)$ and $\mathrm{L}_{\mathrm{w}}(\lambda)$, are more affected than the other apparent optical properties such as downwelling solar irradiance, $\mathrm{E}_{\mathrm{d}}(\lambda)$ and diffuse attenuation coefficient, $\mathrm{K}_{\mathrm{d}}(\lambda)$ 
(Voss et al. 1986; Weir et al. 1994; Weir et al. 1994; Hooker et al. 2003). Large waves have some effect on clear waters with low $K_{d}(\lambda)$ values (Zibordi et al.2009). Though $L_{u}(\lambda)$ and $E_{d}(\lambda)$ may be susceptible to ship shadow, their ratio, remote sensing reflectance $\mathrm{R}_{\mathrm{rs}}(\lambda)$ seems to be less susceptible to the ship shadow effects. The variations in $L_{u}(\lambda)$ are found to vary spectrally with low differences at lower wavelengths and increase towards higher wavelengths (Weir et al. 1995). The errors due to ship shadow are relatively larger under overcast skies as compared to clear skies (Gordon 1985; Voss et al. 1986; Spinrad and Widder 1992).

There are intense flashes of downwelling irradiance in the upper layers of water caused by the wave focusing of sunlight at the surface by the surface waves. The intensity and duration decrease with depth. This effect is likely to be found in calm, clear waters with moderate wind, small waves and clear sky (Dera and Gordon 1968; Snyder and Dera 1970; Dera and Olszewski 1978; Gordon 1985; Dera and Stramski 1986; Stramski and Dera 1988; Zibordi et al. 2009; Hieronymi and Macke 2010). Since phytoplankton activities are mostly in the upper layers, the effect of focusing of light could influence photosynthetic activities (Greene and Gerard 1990). Studies have also been carried out to validate the effects of wave-induced light using radiometer (Gernez and Antoine 2009).

The solar irradiance measured at the surface of water is used as a reference to compute remote sensing reflectance of the surface. These measurements can be taken at the surface of water using a sensor floating at the surface of the water, away from the ship to avoid ship perturbation and ship shadow or at a clear site on a high platform on the ship, avoiding any shadow or reflective surface. Neither method is infallible; however the latter method is often preferred as wave-induced errors are observed in the irradiance values received from the floating sensor.

The radiometers used for the above water measurements, provide only surface parameters. Ship perturbations, sunglint and superstructure are the source of errors for such water measurements (Hooker et al. 2003). Considering all mentioned sources of errors, a free falling profiling underwater light-measuring radiometer was developed that could minimize such errors to a great extent (Smith et al. 1984; Waters et al. 1990). Profiling instruments were earlier deployed using hydrowire (Smith et al. 1984), which gave errors from ship perturbations and superstructure and non-uniform descent rates (Hooker and Maritorena 2000). As a solution to this the instrument was deployed from a boom extending out in the sea from the platform (Hooker and Aiken 1998). Cranes and boom cause additional shadows, they cannot extend beyond their limit to avoid ship shadows and since they are on the ship, pitch and roll can hamper proper deployment and measurements. Winch operations also take considerably more effort and time to complete the operations. The profiling instrument deployed 
on a taut wire from a moored weight at the bottom resulted in uniform tilt and velocity of the instrument (Hooker et al. 2001). The in-water profiling radiometers using latter modes of deployment suffered from the shading effect of the platforms (Voss et al. 1986; Zibordi and Ferrari 1995; Hooker and Maritorena 2000; Hooker et al. 2001; Hooker et al. 2003). Most of the profiling radiometers were tethered with a reinforced cable that carries the power and data, which was avoided in a self-recording hyperspectral radiometer (Desa and Desa 1991; Desa et al 1994). As a remedy to the problem of ship shadow, one of the recommended methods for the deployment of the free falling profiling radiometer was the "fish out" method, taking care to keep the radiometer at a safe distance away from the ship before taking measurements (Waters et al. 1990). Though the source of errors is known, quantifying them and understanding their behavior will permit protocol to process the radiometric data and derive optical parameters with less errors and with required accuracy (Zaneveld et al. 2001; Zibordi et al. 2004).

Free falling radiometer is a better solution to determine the in-water optical parameters avoiding ship shadows and operating with ease in short time. Hydrodynamic studies have shown that movement of a falling cylinder in water column is a highly nonlinear process and can have six trajectory patterns such as straight, spiral, flip, flat, seesaw and combinations of these. Such studies indicate that the trajectories of a rigid cylinder depend on the distance of centre of mass and centre of geometry, drop angle and aspect ratio (length to diameter ratio), initial velocity and physical parameters of the cylinder (Vaidya 2006; Abelev et al. 2007). The orientation of a falling cylinder with respect to the vertical or direction of fall, known as shape-tilting, is proportional to the aspect ratio (length to diameter). The tilt is sensitive to aspect ratio and increases with aspect ratio (Joseph and Liu 1993; Chu et al. 2004; Vaidya 2004, Vaidya 2006). The HyperOCR radiometer is a perfectly designed body with low aspect ratio and the centre of mass is away from the geometric centre or centre of volume, which provides large restoring moment thus enabling vertical trajectories with low tilt (Figure 1). 


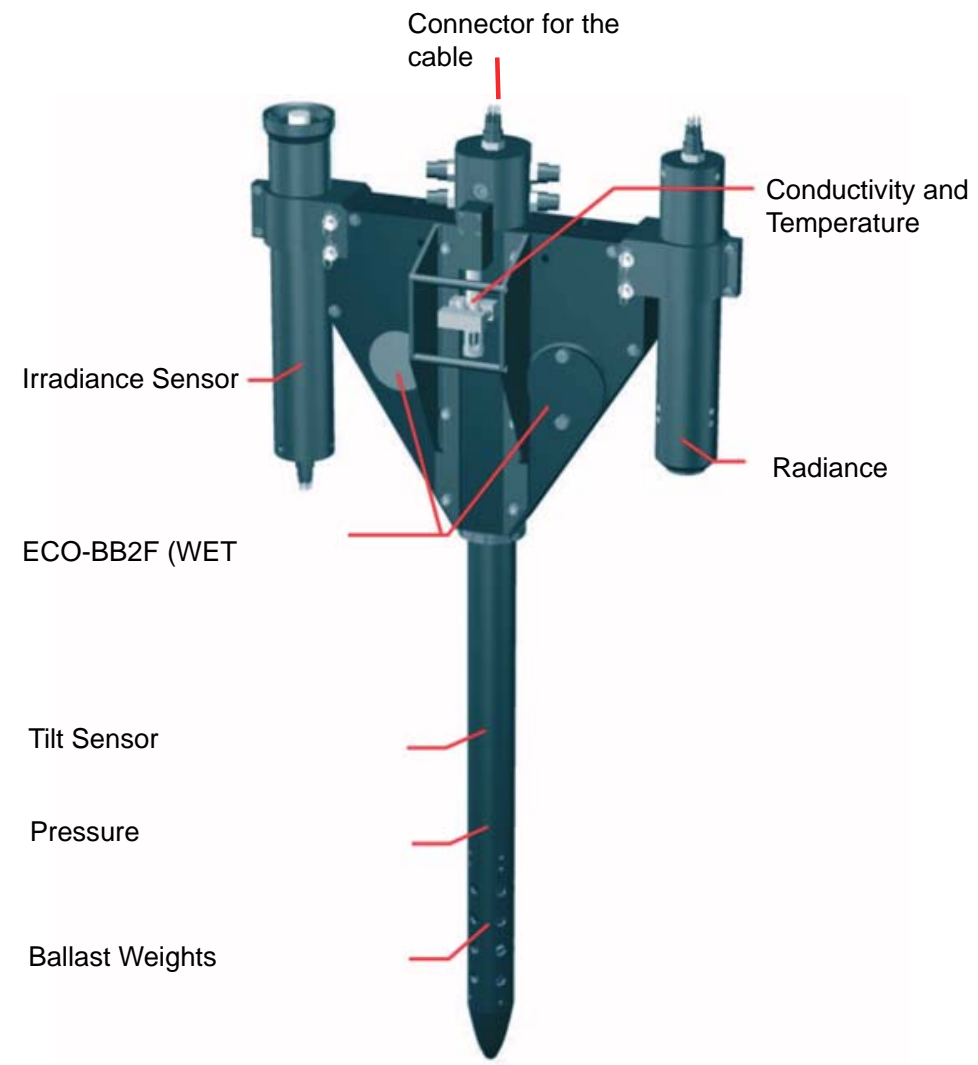

Figure 1: Hyperspectral Radiometer, HyperOCR (http://www.satalantic.com)

We present here the results of the observations of the tilt of the profiling hyperspectral radiometer and the influence of the physical parameters of water. The measurements were carried out during two cruises in the north-eastern Arabian Sea on board research vessel, ORV Sagar Kanya, SK-256 from 9 to 23 February, 2009 and SK-258 from 19 to 29 April, 2009. There have been many studies carriedout on the falling objects in water to understand their behavior during fall and their trajectories. Most of them have been studied in calm water conditions with isotropic properties of temperature, salinity and density and for applications related to a study of the trajectories of the mines (Chu et al. 2002; Holland et al. 2004; Chu et al. 2005; Abelev et al. 2007) and rheological applications (Joseph and Liu 1993; Vaidya 2006). Here we have not attempted any hydrodynamic studies to validate or understand the behavior under the varying physical parameters of the seawater or the design of the radiometer. However it is presumed that the observations and results given here will provide an opportunity to look into these aspects at a later stage to obtain radiometric data from instruments with less error to match the desired accuracy for satellite validation. 


\section{Materials and Method}

The instrument used for measuring underwater light parameters is a free falling hyperspectral radiometer, HyperOCR (Satlantic Inc, Canada, http://www.satlantic.com). The instrument has a ' $T$ ' shaped design with two optical sensors mounted apart on the same horizontal plane on this metal frame (Figure 1). The middle of the ' $\mathrm{T}$ ' section is cylindrical and the bottom is a parabolic nose cone with adjustable ballast weights just above the nose cone. The instrument has fins in the upper section, which provide balance and allow profiling in water during free fall with very low tilt. The free-fall descent rate of the instrument is user-adjustable using appropriate lead weights near the nose cone depending on the water types, Case 1 or Case2. Case 1 waters are those waters dominated primarily by phytoplankton and related colored dissolved organic matter (CDOM) and detritus degradation products (often open ocean) and the rest are Case 2 waters whose optical properties depend on phytoplankton, CDOM, detrital, suspended matter which do not co-vary with phytoplankton (often coastal). Recommended descent rates are from $0.1 \mathrm{~m} / \mathrm{sec}$ to $1.0 \mathrm{~m} / \mathrm{sec}$ in Case- 1 waters and a relatively lower descend rate of 0.1 to $0.3 \mathrm{~m} / \mathrm{sec}$ for Case-II waters. The lower portion of the cylindrical tube with nose cone houses the tilt and pressure sensors, while the upper portion has the temperature and conductivity sensors and electronics and connector for the tethered telemetry cable. The instrument is lowered with this tethered cable, without any external winch wire. In the profile mode of operation, the instrument being lightweight is deployed in "fish out" mode away from the ship, avoiding any ship shadow by a single operator. The reinforced cable carries the power and data to the deck unit and the data is acquired on a computer. The commonly used hyperspectral optical sensors are irradiance and radiance and have a spectral range of $350-800 \mathrm{~nm}$. The irradiance sensor measures the downwelling irradiance while the radiance sensor is used to measure the upwelling radiance.

The instrument is deployed in "fish out" mode positioning the boat to keep the radiometer well under the sun, with bow-stern perpendicular to the position of sun, allowing the ship or boat to drift away from the radiometer, so that it does not come under the shadow after it is deployed. Most of the measurements are taken at noon with low zenith angle, which however adds to the disadvantage, as winds are usually high around noon period and the surface waters are always rough with swells and waves. In the Arabian Sea, when the instrument was hauled up after every cast, the instrument often surfaced far away $(\sim 50 \mathrm{~m})$ from the ship due to the currents, which was not so when it was deployed in the estuary. After the cast, when it is brought up with the help of the tethered cable, the turbulence at the surface due to waves, does not allow the radiometer to be in vertical position even when the 
cable is kept slightly slack. This drag keeps the radiometer incline to the vertical and close to the surface. Being a free falling instrument, the radiometer is deployed when required by releasing the cable. Under this condition, the radiometer often commences the dives from an inclined plane and attains the vertical position with low orientation after having traversed through some depths during this period (Figure 2). Thus the data from the radiometer is tainted with large tilt angles from the surface to a few meters below. The radiometer thereafter maintains low tilt as it descends vertically. Since data beyond the tolerance limit of tilt, which is taken as 5 degrees, is discarded during post processing, we often miss critical data from the surface layer. As observed from the experience of deploying the free falling radiometer, this seems to be an inherent drawback, a price that needs to be paid for avoiding the ship shadow.

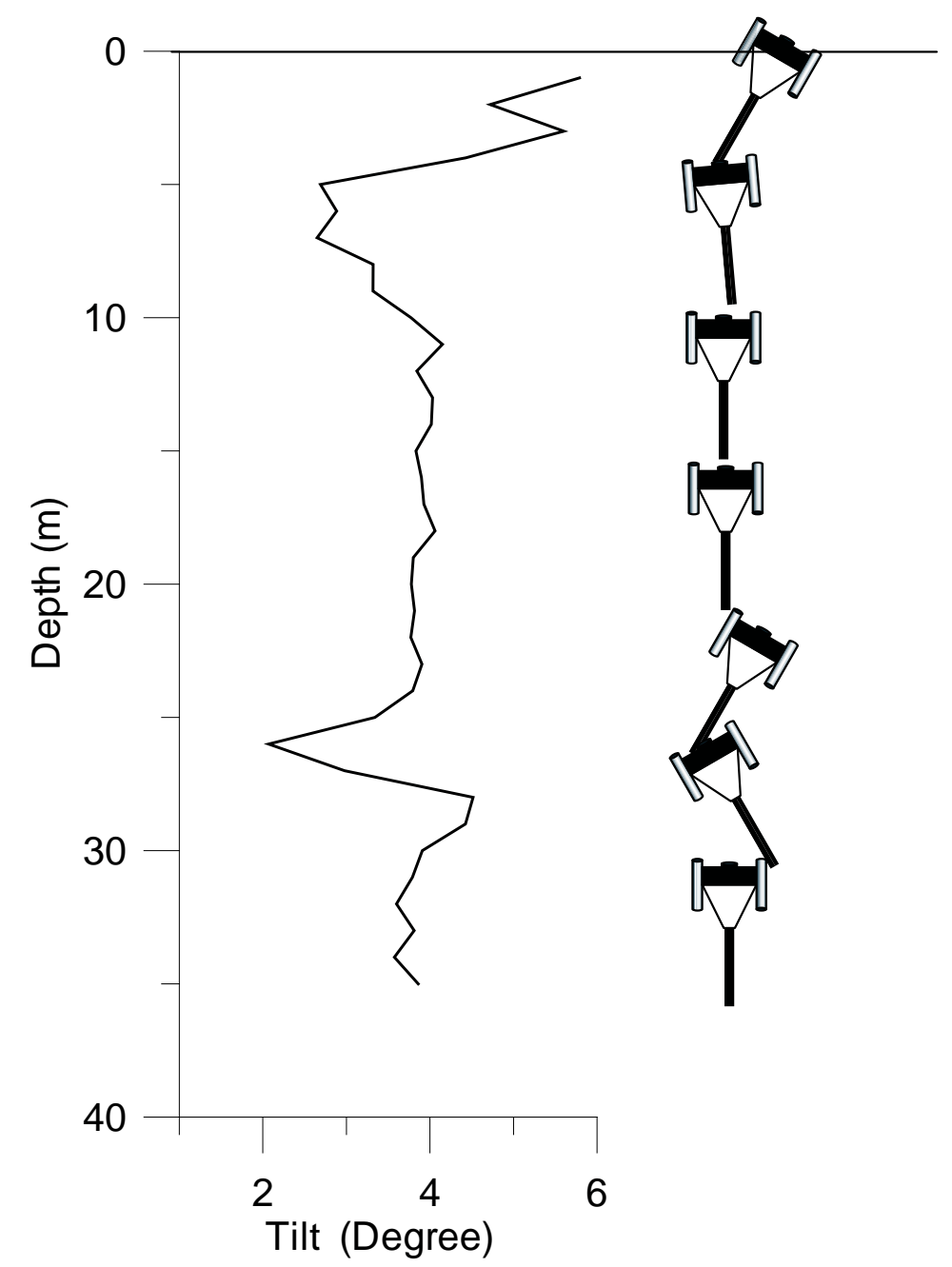

Figure 2: Pictorial view of the radiometer at the surface before falling and the orientation in the water 


\section{Results}

The average tilt of the radiometer in water observed during the two cruises SK256 and SK-258 are $4.2^{\circ}\left( \pm 1.26^{\circ}\right), 3.05^{\circ}$ degree $\left( \pm 1.02^{\circ}\right)$ respectively. Apart from the tilt of the instrument at the surface layers, one of the observable features has been the variation in the tilt wherever it encountered a change in the density of the water (Figure 3). Since the radiometer was also equipped with sensors for temperature and conductivity, the density (Sigma-t) was computed using these parameters.
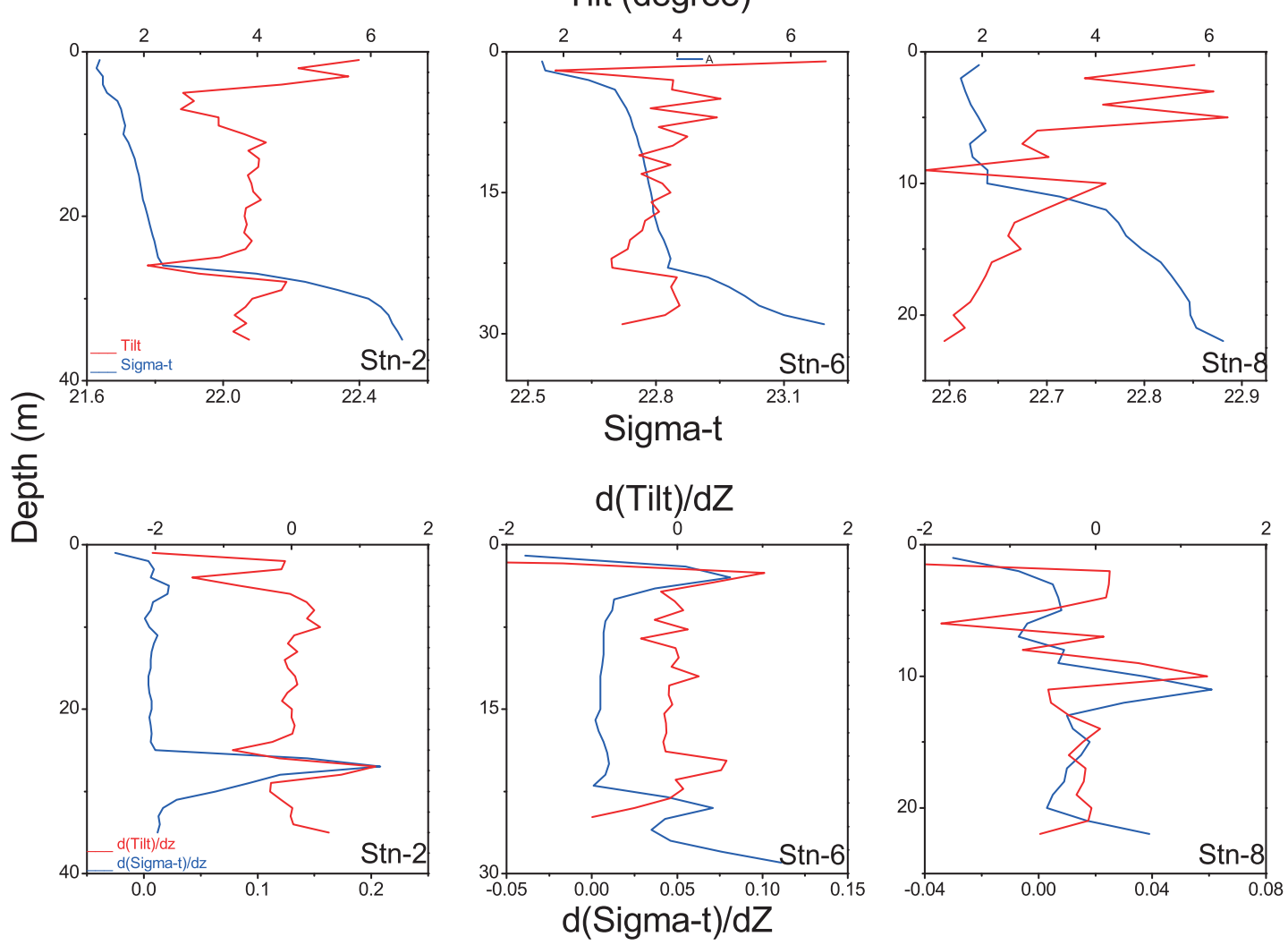

Figure 3: The top figure shows the variations of tilt of the radiometer and the density of water. The figure below is the derivative of tilt and density with respect to the depth. (Cruise SK-258, Stations 2, 6 and 8).

Density stratification in the ocean is an important feature that influences biological, chemical, and physical processes. The stratifications exhibit changes in pycnocline, thermocline and halocline. Indicators of stratifications such as barrier, mixed and isothermal layers are found to vary with season in the Arabian Sea, with mixed layer depth varying between 20 to $70 \mathrm{~m}$, maximum being observed during the period of southwest monsoon (Sreenivas et al. 2008). These regions are in the upper layers and are the outcomes of vigorous turbulent mixing processes. At times surface layer 
temperature inversions are observed in the coastal waters of the eastern Arabian Sea during winter (Thadathil et al. 2008). At the interface of change of density, there is an appreciable negative tilt and thereafter the instrument steadies itself and descends vertically. During winter, at times the tilt also mimics the density profiles, often increasing with depth (Figure 4).

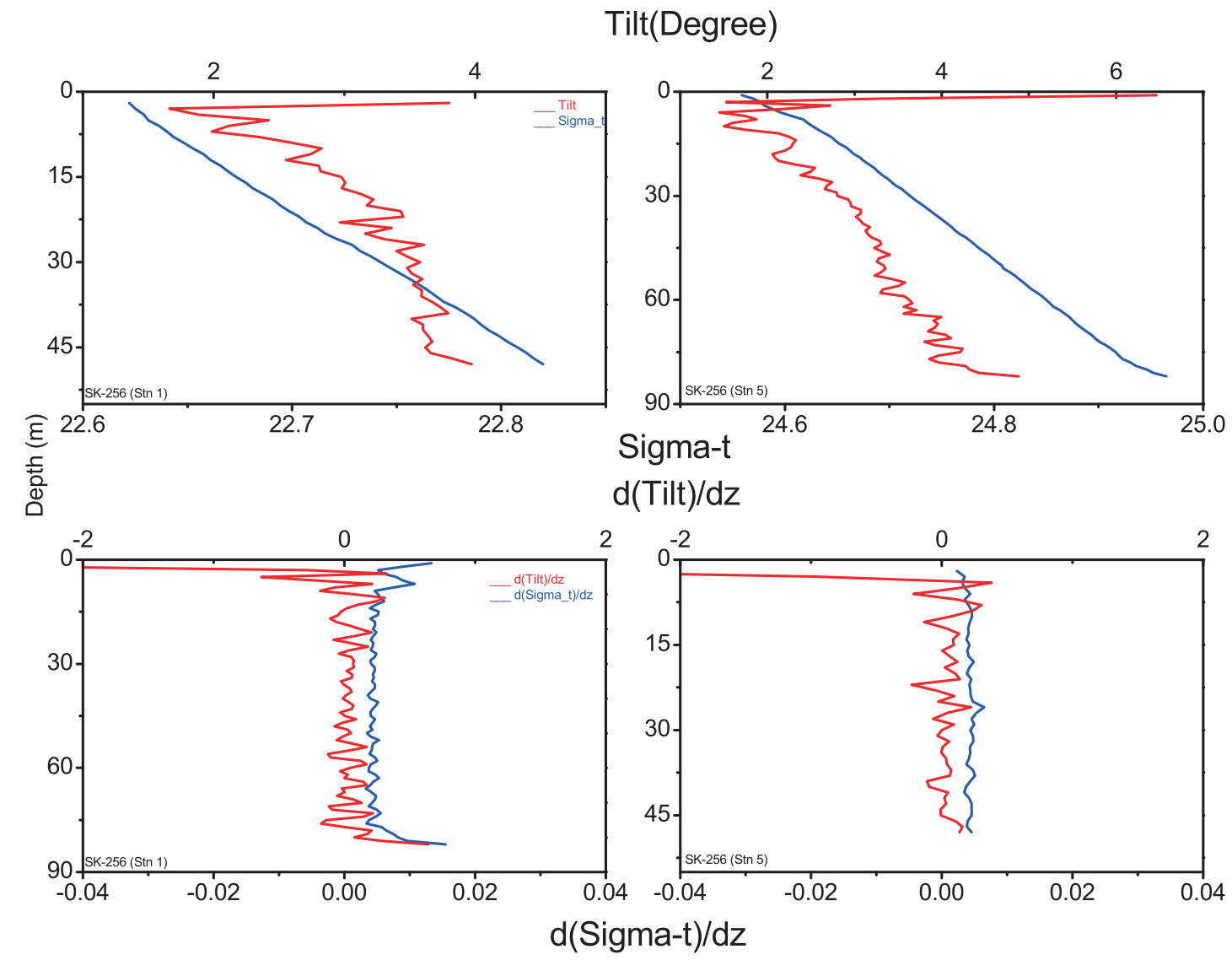

Figure 4: The variation in tilt is found to follow the density of water

We did not see any similar correlating features in the velocity or acceleration data of the radiometer at the interfaces of change in the density (Figure 5). 

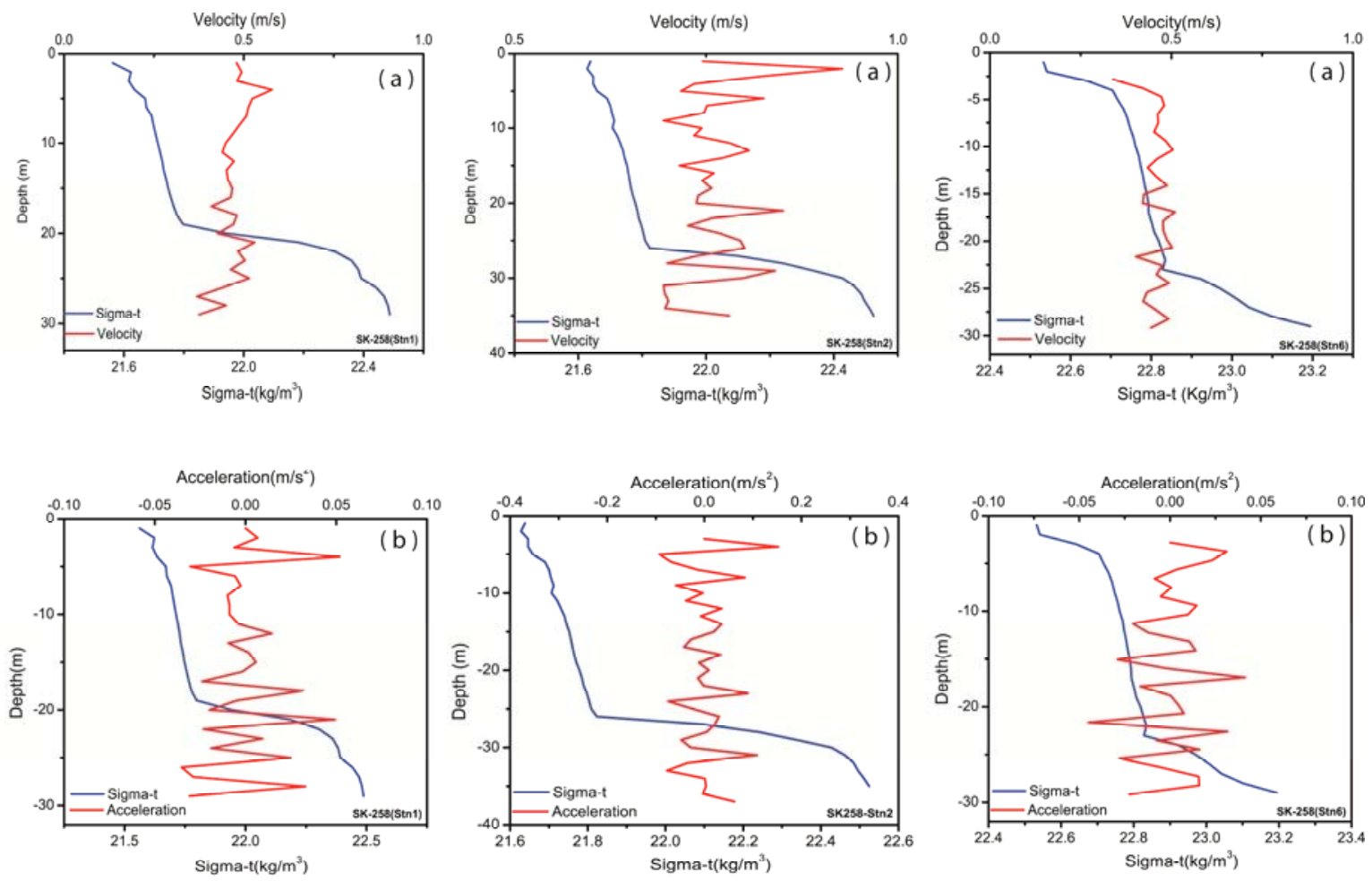

Figure 5: (a) Velocity and (b) Acceleration of the radiometer and the density variation of water. No appreciable change in velocity and acceleration is observed.

These variations of tilt due to change in the density are more pronounced in stratified waters in the upper layer. In less stratified waters in the upper layers as observed during winter (Cruise SK-256), the profile is found to be smooth with very minimal variations in density and tilt (Figure 6). The sharp variations in tilt during its dive in the upper layer is however omnipresent. 
Tilt (degree)

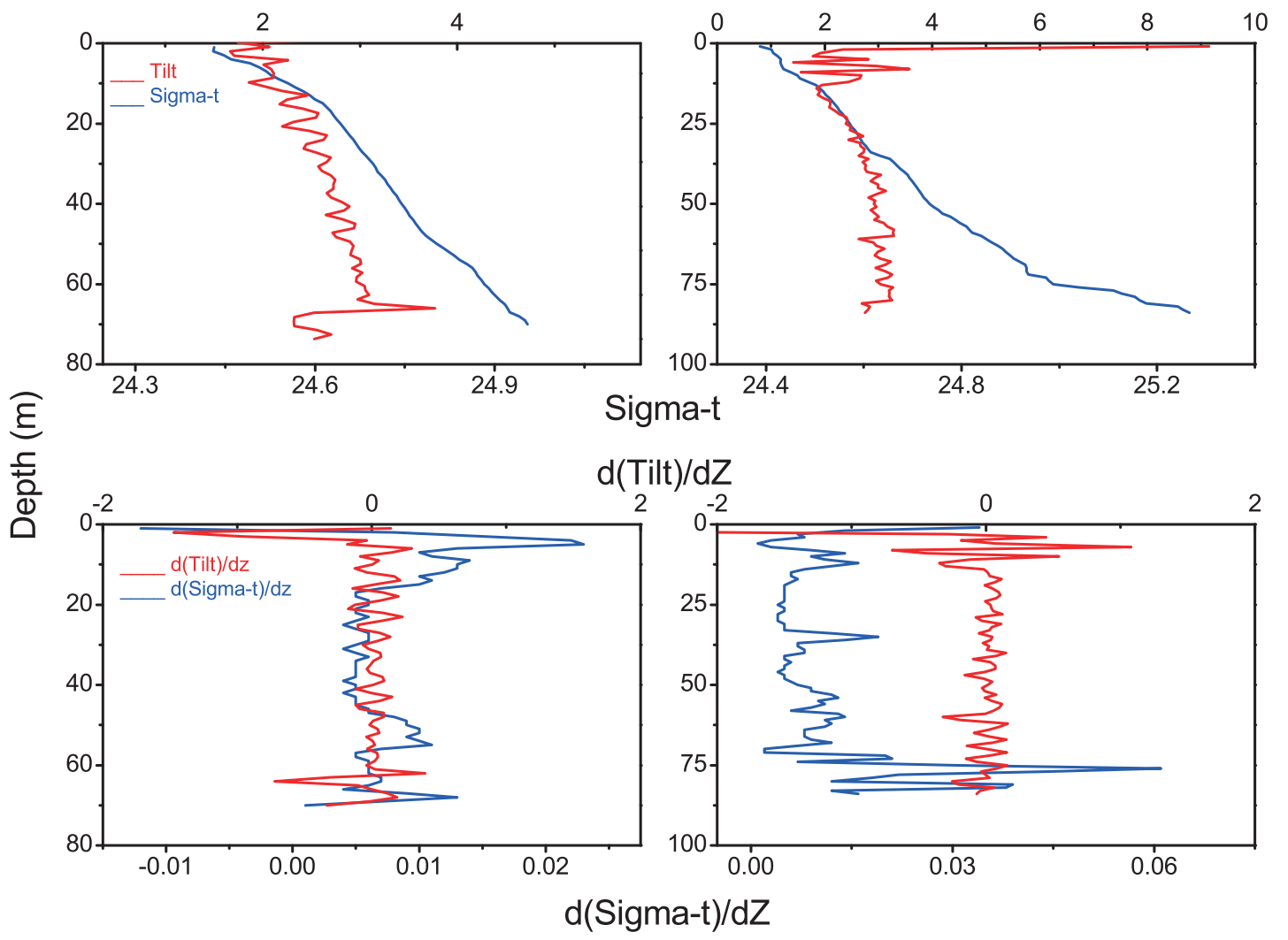

Figure 6: The top figure shows the variations of tilt of the radiometer and the density of water during the winter, when the stratification is found to be less compared to the summer. The figure below is the derivative with respect to the depth. (Cruise SK-256).

\section{Discussion and Suggestions}

The above water parameters such as water leaving radiance, $\mathrm{L}_{\mathrm{w}}(\lambda)$ have to be obtained by extrapolating the in-water measurements to the surface (Mueller and Austin 1995; Mueller et al. 2003; Friedman et al. 2003). The above water parameters obtained by extrapolation is done under various assumptions such as the invariance of the attenuation with depth and so the values obtained could have errors. There would be fewer errors if the optical data could be obtained as close to the surface within tolerable limits of tilt (in our case we chose 5 degree). Apart from the body geometry, the body motion is also affected by environmental conditions such as waves and currents during the fall (Kim et al. 2002; Friedman et al. 2003). Since there is always turbulence in the upper layers it is observed that the tilts are unavoidable in the upper layers, and hence these data cannot be considered. The data within this layer is obtained by using the information on data of the layers below it, and extrapolating to the surface. 
Since our objective is to obtain the water leaving radiance within the desired accuracy as required for the ocean color satellite validation (Hooker et al. 2003) it is essential to measure the optical parameters as close to the surface with less error. In order to achieve this, we need to take care of all other constraints while deploying the radiometer such as ship shadow, drift and sun position. Since the loss in the upper layer of water was attributed to the slow dive at an inclined plane, we thought it prudent to avoid the "fish out" method and find a method that will allow the radiometer to dive vertically from the surface. Hydrodynamic studies on a falling cylinder have shown that the orientation is influenced by the mode of release and its influence is observed only in the upper surface layers of the water (Abelev et al. 2007). To minimize this effect in the initial stages, an extended boom with pulley arrangement and a marine release hook arrangement was used for the trial (Figure 8). The boom extended to about $2 \mathrm{~m}$ from the boat and was placed firmly at the gunwale of the boat. The marine hook was hung over the water with a rope going over the pulley whose other end over the pulley was held firm. The radiometer was hung from the marine hook. Pulling the lever vertical by a thin rope fastened to it from the boat operates the marine hook release mechanism and releases the radiometer in water. The radiometer is positioned just above the water and it stays vertical. Assuming that the pressure sensor does not always give the correct depth at the surface, pressure tare to determine the depth offset and get the right depth from the surface is performed after adjusting the height of radiometer to be just above water, hence there is less ambiguity in determining the depth from the surface. The tethered radiometer cable is held loose on the deck of the boat before deployment. Thereafter the data logging starts and the radiometer is released from the hook by pulling the lever of the marine hook. The radiometer dives vertically and the cable is paid out with less tension, keeping in tandem with the descent of the radiometer while taking care to release only the required amount of cable. The operation of the release mechanism lever does not demand any effort and the radiometer can be deployed with ease. After the radiometer reaches the required depth of at least $1 \%$ of light level, radiometer is hauled up with the reinforced cable of the radiometer and readied for the next cast.

The disadvantage of this method is that it suffers from the shadow of the boat and boom. Extending the boom up to $10 \mathrm{~m}$ and keeping the structure very narrow can circumvent the problem of ship shadow. We had a trial using the boom and marine hook under very rough conditions at the mouth of the Zuari estuary, Goa, during the monsoon period of July 2010. The results were very encouraging and we were able to get the surface data with less tilt when compared to the conventional "fish out" method of deployment (Figure 7). 


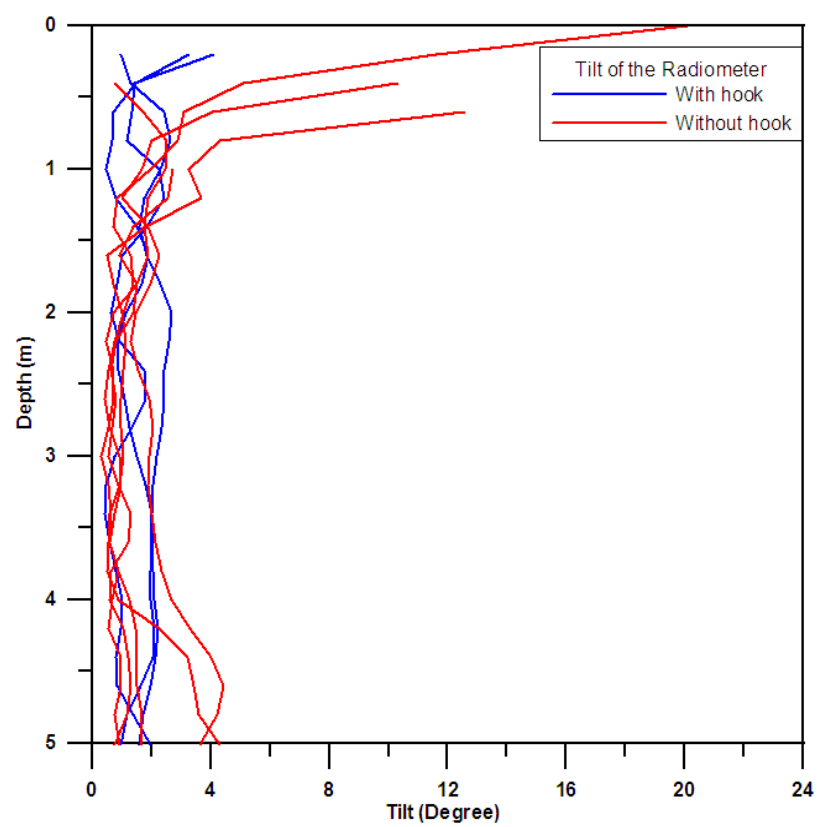

Figure 7: The results of the trials of deploying the radiometer using the boom and release hook and the normal "fish out" method.

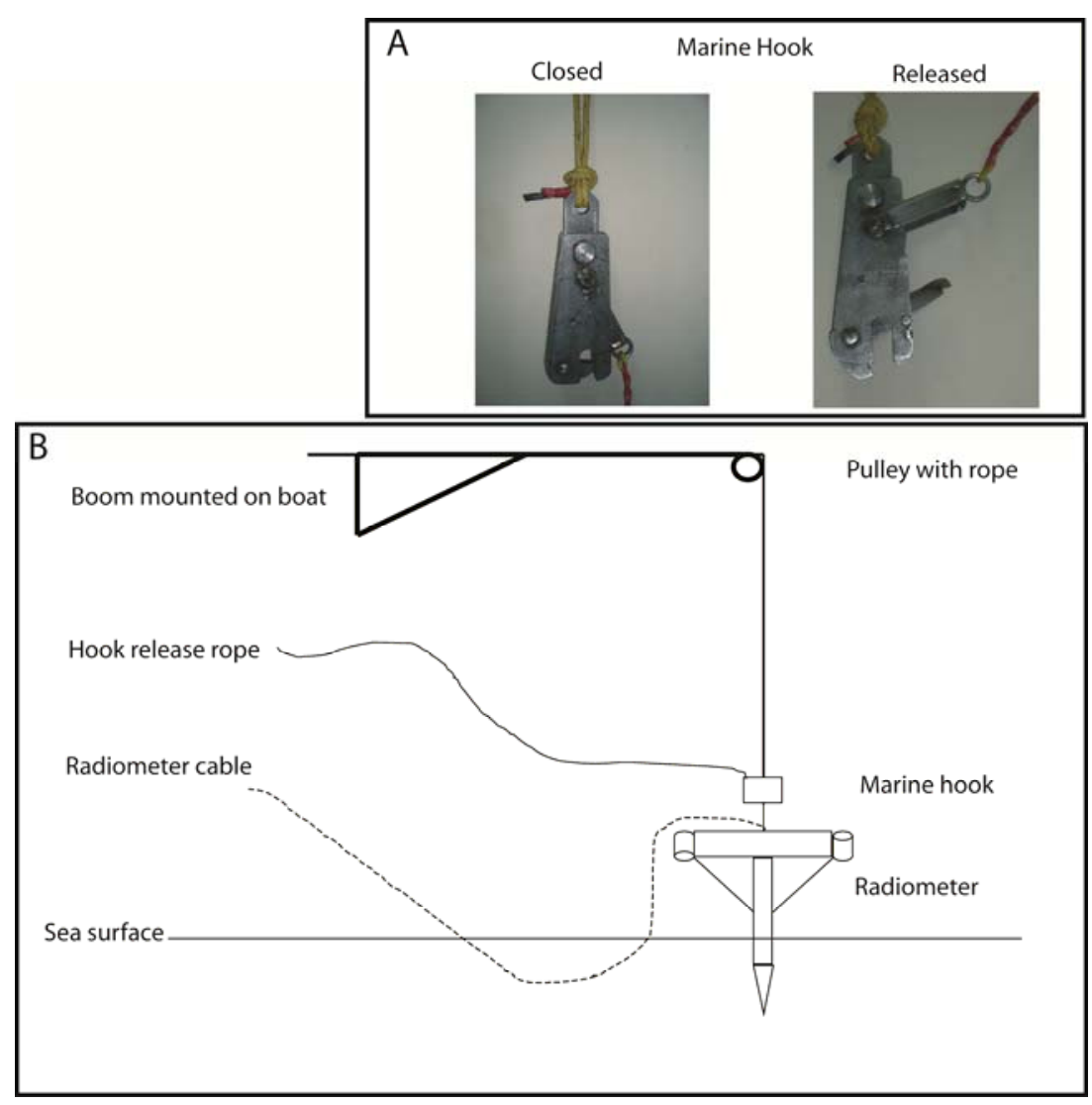

Figure 8:Deployment of radiometer using boom and marine hook to reduce tilt of the radiometer at the surface. 
The trials have confirmed that the tilt observed in the surface layer is primarily due to mode of deployment and turbulence of water, which does not allow the radiometer to stay vertical during the dive. Hence a method is suggested that will keep the radiometer afloat on the surface of water maintaining vertical position and then releasing the radiometer to dive vertically down. Whatever the method adopted, care should be taken that no shadow is cast over the instrument, with the release being smooth without a jerk so as to allow the instrument to fall freely and descend without appreciable tilt.

Here we also place some suggestions that could help in improving the performance of the hyperspectral radiometer. We have observed that often after diving through some depth beyond 80 $\mathrm{m}$, there is a large tilt in the instrument. These effects could not be attributed to the change in the density. The plausible reason for this is attributed to the tethered cable, which produces negative buoyancy due to the air trapped in the jacket of the cable. The solution to this problem is do away with tethered cable and a have self-recording feature in the radiometer (Desa and Desa 1991; Desa et al. 1994). The stability of the radiometer could probably be improved by changing the shape of the nose cone. The radiometer has cone shaped nose ends, and it has been reported that the trajectories also depend on the shape of the cylinder nose ends and blunt shape is found to be hydrodynamically more stable (Abelev et al. 2007).

\section{Conclusion}

Measurements using the free falling profiling hyperspectral radiometer require utmost care to obtain optical parameters with minimum errors avoiding ship shadow and tilt of the radiometer during measurements. We have identified the two sources that affect the tilt of a perfectly balanced profiling instrument, one from the upper surface layers, which is attributed to the surface environmental conditions and mode of deployment of the instrument and the second due to the density stratifications. The depth at which the tilt occurs depend on density stratification or mixed layer, this depth will vary with water types and season. Since large tilt is observed at the density stratification, shallow mixed layer will cause errors closer to the surface, while effect of deep mixed layer beyond penetration or optical depth will cause relatively much less error. It was concluded using a prototype model, that the tilts observed at the surface layers could be minimized using a simple marine hook release mechanism. Hence it is proposed that a well-designed mechanism deploying the radiometer away from the ship shadow and avoiding self-shadows could get rid of the tilt observed in the layers close to the surface. This will provide the much required radiometric data with less tilt of the radiometer and errors at the surface of water. 


\section{Acknowledgement}

We are thankful to the Director, National Institute of Oceanography for his encouragement and support. The work was carried out under the project on bio-optical measurements of the coastal waters, funded under SATCORE program of Indian National Centre for Ocean Information Services, India. The authors are indebted to the Ministry of Earth Sciences for providing the research vessel, ORV Sagar Kanya. Thanks to Miss Amanda Fernandes all the help rendered in preparing the manuscript.

\section{References}

Aas, E. and O. B. Korsb. 1997. Self-shading effect by radiance meters on upward radiance observed in coastal waters. Limnology and Oceanography. 42:968-974.

Abelev, A. V., P. J. Valent, and K. T. Holland. 2007. Behavior of a large cylinder in free fall through water. IEEE J. Ocean. Eng. 32(1):10-20.

Chu, P. C., A. Gilles, C. Fan. 2005. Experiment of falling cylinder through the water column. Experimental Thermal and Fluid Science. 29(5):555-568.

Chu, P. C., C. W. Fan, A. D. Evans, A. Gilles. 2004. Triple coordinate transforms for prediction of falling cylinder through the water column. Journal of Applied Mechanics. 71:292-298.

Chu, P.C., A. F. Gilles, C. W. Fan, J. Lan, P. Fleischer. 2002. Hydrodynamics of falling cylinder in water column. Advances in Fluid Mechanics. 4:163-181.

Dera, J., and D. Stramski. 1986. Maximum effects of sunlight focusing under a wind-disturbed sea surface. Oceanologia. 23:15-42.

Dera, J., and H. R. Gordon. 1968. Light field fluctuations in the photic zone. Limnology and Oceanography.13:697-699.

Dera, J., and J. Olszewski. 1978. Experimental study of short-period irradiance fluctuations under an undulated sea surface. Oceanologia. 10:27-49.

Desa, E.S., and B. A. E. Desa. 1991. Design of an in-water spectrograph for radiance measurements in the ocean. Optical Engineering. 30:1576-1582.

Desa, E.S., E. Desa, R.G. Prabhudesai, T. Suresh. 1994. A fast scanning high-resolution in-water spectroradiometer. OCEANS '94'. 3:13-16.

Friedman, A., G. Zilman and T. Miloh. 2003. Dynamics of a body falling in waves. 18th IWWWFB, Le Croisic, France, 2003.

Gernez P., and D. Antoine. 2009. Field characterization of wave-induced underwater light field fluctuations. J. Geophys. Res. 114:C06025.

Gordon, H. R. 1985. Ship perturbation of irradiance measurements at sea. Part 1: Monte Carlo simulations. Applied Optics. 24:4172-4182. 
Gordon, H. R., and K. Ding. 1992. Self-shading of in-water optical instruments. Limnology and Oceanography. 37:491-500.

Greene, R. M., and V. A. Gerard. 1990. Effects of high-frequency light fluctuations on growth and photoacclimation of the red alga Chondrus crispus. Marine Biology. 105:337-344.

Hieronymi, M., and A. Macke. 2010. Spatiotemporal underwater light field fluctuations in the open ocean. J. Europ. Opt. Soc. Rap. Public. 10019:5.

Holland, K. T., A. W. Green, A. V. Abelev, and P. J. Valent. 2004. Parameterization of the in-water motions of falling cylinders using high-speed video. J. Exp. Fluids. 37:690-700.

Hooker, S. B., and S. Maritorena. 2000. An evaluation of oceanographic radiometers and deployment methodologies. J. Atmos. Oceanic Technol. 17:811-830.

Hooker, S. B., and W. E. Esaias. 1993. An overview of the SeaWiFS project. EOS Trans. Amer. Geophys. Union. 74:241-246.

Hooker, S. B., G. D. Zibordi, J. F. Berthon, D. D’Alimonte, S. Maritorena, S. McLean, and J. Sildam. 2001. Results of the Second SeaWiFS Data Analysis Round Robin, March 2000 (DARR-00). NASA Tech. Memo 2001-206892, 15:71. S. B. Hooker and E. R. Firestone, Eds.,NASA Goddard Space Flight Center, Greenbelt, Maryland.

Hooker, S. B., G. Lazin, G. Zibordi, and S. McLean. An evaluation of above and in water methods for determining water-leaving radiances. J. Atmos. Oceanic Technol. 19:486-515.

Hooker, S. B., G. Zibordi, D. van der Linde, D. D'Alimonte, J. F. Berthon and J. Brown. 2002. Tower perturbation measurements in above-water radiometry. NASA TM 2002-206892, v. 23:35. S.B.Hooker and E.R.Firestone, Eds., NASA Goddard Space Flight Center, Greenbelt, Maryland, 2003.

Hooker, S. B., and J. Aiken. 1998. Calibration evaluation and radiometric testing of field radiometers with the SeaWiFS Quality Monitor (SQM). J. Atmos. Oceanic Technol. 15:9951007.

Joseph, D. D., and Y. J. Liu. 1993. Orientation of long bodies falling in a viscoelastic liquid. Journal of Rheology. 37:961-983.

Kim, Y., Y. Liu, D. K. P. Yue. 2002. Motion dynamics of three-dimensional bodies falling through water. $17^{\text {th }}$ International Workshop on Water Waves and Floating Bodies, Cambridge, UK, 2002.

Mueller, J. L., A. Morel, R. Frouin, C. Davis, R. Arnone, K. Carder, Z. P. Lee, R.G. Steward, S. Hooker, C. D. Mobley, S. McLean, B.N. Holben, M. Miller, C. Pietras, K. D. Knobelspiesse, G. S. Fargion, J. Porter, and K. Voss. 2003. Ocean Optics Protocols for Satellite Ocean Color Sensor Validation, Revision 4, Volume III: Radiometric Measurements and Data Analysis Protocols NASA/TM-2003-211621/Rev4-Vol. III, 2003.

Mueller, J. L., and R. W. Austin. 1995. Ocean Optics Protocols for SeaWiFS Validation, Revision 1. NASA Tech. Memo. 104566, 25:67. S.B. Hooker, E.R. Firestone and J.G. Acker, Eds. NASA Goddard Space Flight Center, Greenbelt, Maryland, 1995. 
Smith, R.C., C.R. Booth and J. L. Star1984. Oceanographic bio-optical profiling system. Applied Optics. 23:2791.

Snyder, R. L., and J. Dera. 1970. Wave-induced light-field fluctuations in the sea. J. Opt. Soc. Amer. 60:1072-1079.

Spinrad, R.W. and E. A. Widder.1992. Ship shadow measurements obtained from a manned submersible. SPIE Proceedings, 1750:372-383.

Sreenivas, P., K. V. K. R. K Patnaik and K. V. S. R. Prasad. 2008. Monthly Variability of Mixed Layer over Arabian Sea Using ARGO Data. Marine Geodesy. 31:17 - 38.

Stramski, D., and J. Dera. 1988. On the mechanism for producing flashing light under a wind disturbed water surface. Oceanologia. 25:5-21.

Thadathil, P., P. Thoppil, R.R. Rao, P. M. Muraleedharan, Y. K. Somayajulu, V. V. Gopalakrishna, R. Murthugudde, G. V. Reddy, and C. Revichandran. 2008. Seasonal variability of the observed barrier layer in the Arabian Sea. Journal of Physical Oceanography. 38:624-638.

Vaidya, A. 2004. Orientation of Rigid Bodies Freefalling in Newtonian and Non-Newtonian Fluids. Ph.D. thesis, Department of Mechanical Engineering, University of Pittsburgh.

Vaidya, A. 2006. Observations on the Transient Nature of Shape-tilting Bodies in Polymeric Fluids, Journal of Fluids and Structures. 22(2):253-259.

Voss, K. J., J. W. Nolten, and G. D. Edwards. 1986. Ship shadow effects on apparent optical properties. Ocean Optics VIII, SPIE. 637:186-190.

Waters, K. J., R. C. Smith, and M. R. Lewis. 1990. Avoiding ship induced light- field perturbation in the determination of oceanic optical properties. Oceanography. 3:18-21.

Weir, C. T., D. A. Siegel, A. F. Michaels, and D. W. Menzies. 1994. In situ evaluation of a ship's shadow. Ocean Optics VIII, M. A. Blizzard, Ed. 637:186-190.

Weir, C. T., D. A. Siegel, D. W. Menzies, and A. F. Michaels. 1995. In situ evaluation of a ship's shadow. Case Studies for SeaWIFS Calibration and Validation, Part 3, NASA Tech. Memo. 104566, S. B. Hooker, E. R. Firestone, and J. G. Acker, Eds 27:25-33.

Zaneveld, J. R. V., E. Boss, and A. Barnard. 2001. Influence of surface waves on measured and modeled irradiance profiles. Applied Optics. 40:1442- 1449.

Zibordi, G., and M. Ferrari. 1995. Instrument self-shading in under-water optical measurements: Experimental data. Applied Optics. 34:2750-2754.

Zibordi, G., Berthon, J. F. and D'Alimonte, D. 2009. An evaluation of radiometric products from fixed-depth and continuous in-water profile data from moderately complex waters. Journal of Atmospheric and Oceanic Technology, 26:91-106.

Zibordi, G., D. D'Alimonte, and J. F. Berthon. 2004. An evaluation of depth resolution requirements for optical profiling in coastal waters. J. Atmos. Oceanic Tech 21:1059- 1073. 